\title{
Investigation of the expressions of MMPs and TIMPs between isogeneic and allogeneic rat aortic transplantation
}

\author{
SI-DONG WEI ${ }^{1}$, DING CAO $^{2}$, XUE-SONG XU $^{2}, \mathrm{HE} \mathrm{BAI}^{2}, \mathrm{HAO} \mathrm{WU}^{2}$ and MENG-HAO WANG ${ }^{2}$ \\ ${ }^{1}$ Department of Hepatobiliary Surgery, People's Hospital of Zhengzhou, Southern Medical University, \\ Zhengzhou, Henan 450003; ${ }^{2}$ Department of Hepatobiliary Surgery, Second Affiliated Hospital of \\ Chongqing Medical University, Chongqing 400010, P.R. China
}

Received November 2, 2017; Accepted February 13, 2018

DOI: $10.3892 / \mathrm{mmr} .2018 .8684$

\begin{abstract}
The present study investigated the effects of matrix metalloproteinases (MMPs) and tissue inhibitor of metalloproteinases (TIMPs) in transplantation-associated arteriosclerosis by observing their expression in transplanted aortas in rats. Allogenic and isogenic abdominal aortic transplantations were performed and grafts were removed from the recipients at the designated time points (day 7, 14, 28 and 56 post transplantation). Hematoxylin and eosin staining, immunohistochemistry, immunofluorescence and western blot analysis were used to evaluate the grafts. Significant proliferation of the intima was observed in the allogenic transplantation groups $(\mathrm{P}<0.05)$. The expressions of MMPs and TIMPs in the allografts were significantly increased compared with the isografts, and the suppression of MMP2 in allografts reduced injury after transplantation. The present study concluded that the imbalance of MMPs and TIMPs led to the disturbance of synthesis and the degradation of the extracellular matrix and it may represent a key cause of chronic rejection.
\end{abstract}

\section{Introduction}

Transplantation is the most effective and permanent treatment for end-stage organ failure but this option is limited because of the acute and chronic rejection of most grafts. Chronic rejection, which is also known as chronic allograft dysfunction, is the main obstacle to achieving the long-term graft survival. Chronic allograft dysfunction shows different histological appearances in various organs. However, their common pathological features are considered to be transplanted arteriosclerosis (TA) (1). Various lesions of vessel wall lead to vascular smooth muscle cells (SMC) activation. Activated SMCs migrate

Correspondence to: Dr Meng-Hao Wang, Department of Hepatobiliary Surgery, Second Affiliated Hospital of Chongqing Medical University, 76 Linjiang Road, Chongqing 400010, P.R. China E-mail: jameswang510@foxmail.com

Key words: aortic transplantation, matrix metalloproteinases, tissue inhibitor of metalloproteinases from the media into the intima by proliferating and elaborating cytokines and extracellular matrix (ECM) proteins, eventually resulting in luminal narrowing and vascular dysfunction (2). Matrix metalloproteinases (MMPs) represent the family of zinc-containing enzymes which degrade the ECM and connective tissue proteins (3). The proteolytic effects of MMPs play an important role in vascular remodeling, cellular migration, and in managing of ECM proteins and adhesion molecules $(3,4)$. Under the physiological state, MMPs interact with particular ECM components and their activities are regulated at the transcription level. Endogenous tissue inhibitor of metalloproteinases (TIMPs) can prevent excessive degradation of ECM. An imbalance between MMPs and TIMPs could lead to the elevation of MMPs activity that may cause pathological changes in the vessel wall structure. The aim of our study is to reveal their function by dynamically observing their expression and blocking their function in allogenic transplantation groups.

\section{Materials and methods}

Animal grouping. In total, 120 Wistar and 40 Sprague Dawley (SD) rats (male, 200-230 g) were obtained from Animal Experiment Center of Chongqing Medical University and housed under specific pathogen-free (SPF) conditions which were maintained at $24 \pm 2^{\circ} \mathrm{C}$ and a 12 -h light/dark cycle. They were randomly divided into 4 groups, which including: i) Control group: Sham operations were performed as control; ii) isogenic group: Orthotopic abdominal aortic transplantation from Wistar to Wistar rats was performed; iii) allogenic group: Orthotopic abdominal aortic transplantation from SD to Wistar rats was performed; and v) allogenic+SB-3CT treated group: Orthotopic abdominal aortic transplantation from SD to Wistar rats was performed. The recipients received SB-3CT (a potent inhibitor of MMP-2, $25 \mathrm{mg} / \mathrm{kg}$ ) once daily for 28 days in the post-transplantation period.

All animals' care and experimental protocols were complied with the National Institutes of Health guide for the care and use of Laboratory animals (NIH Publications no. 8023, revised 1978) and the Animal Management Rules of the Ministry of Health of China and all experimental designs were approved by the Animal Care and Use Committee of Second Affiliated Hospital of Chongqing Medical University (Chongqing, China). 
Orthotopic abdominal aortic transplantation

Donor operation. The donors were anesthetized by intraperitoneal injection of $1 \%$ pentobarbital $(50 \mathrm{mg} / \mathrm{kg})$ and then they were placed in the supine position on the operating board with their abdomen shaved and scrubbed by povidone-iodine. After a long midline abdominal incision, the abdominal aorta was isolated from the level of the left renal vein to the bifurcation via careful blunt and sharp dissection. All aortic branches between the renal arteries and bifurcation were ligated with the help of 9-0 suture (DE FORCE, Shanghai Medical Instruments Co., Ltd, Shanghai, China). This section with an average length of 1.5 to $2.0 \mathrm{~cm}$ can serve as the donor material. Before separating the aorta, two sutures were tied right near the ligations (below renal artery and above bifurcation). Finally, the grafts were fully rinsed with heparin sodium $(200 \mathrm{U} / \mathrm{ml})$ and then kept in lactated Ringer's solution in $4^{\circ} \mathrm{C}$ refrigerator.

Recipient operation. Recipients were anesthetized with isoflurane (4\% for induction and $2 \%$ for maintenance) by continuous inhalation anesthesia. After the midline abdominal incision, the intestines were retracted to the right side to expose the retroperitoneum and then the intestines were covered with a square of wet sterile gauze. The fatty tissue around the aorta and the inferior vena cava (IVC) was gently removed using fine tissue forceps (Jinzhong, Shanghai Medical Instruments Co., Ltd) and the infrarenal aorta was dissected from the IVC to create enough space for two artery clamps. Every other vessel branches were ligated during the dissection.

Two clamps (below renal artery and above bifurcation) were used to stop blood flow before removing the aorta. The cut ends were rinsed with heparinized saline $(200 \mathrm{U} / \mathrm{ml})$ and the graft was end-to-end anastomosed to the recipient through interrupted 9-0 suture (DE FORCE). Before releasing the distal clamp, the proximal clamp was carefully removed to avoid blood leakage at the suture lines. The graft was immediately filled with blood and visible pulsation could be observed. Finally, the intestines were put back into their original place and the abdominal incision was sutured with 4-0 nylon (Ethicon, Inc., Cincinnati, OH, USA). Then $1 \%$ of lidocaine was added to establish analgesia and the rats were kept on a warming pad until they fully recovered from the anesthesia.

Histopathological examination. The transplanted aortas were removed on the 7th, 14th, 28th and 56th day after the operation. All tissues were fixed in $4 \%$ paraformaldehyde for $24 \mathrm{~h}$ immediately after surgical resection and then embedded in paraffin. The tissue sections $(5 \mu \mathrm{m})$ were heated at $60^{\circ} \mathrm{C}$ for $1 \mathrm{~h}$ then dewaxed and rehydrated by immersion in dimethylbenzene and ethanol series and hematoxylin and eosin (H\&E) staining was performed. H\&E statistics were conducted using a method described by Wiebke and colleagues (5). Generally, digitized histological photographs were analyzed and relative thickness (\%) of the intima was calculated by the equation $\mathrm{R}=$ [area (intima)/area (media) $\mathrm{x} 100 \%$, for each group, a mean value of the 4 samples were calculated.

For immunohistochemistry, an antigen-retrieval technique consisting of heating in a citrate water bath at $95^{\circ} \mathrm{C}$ for $20 \mathrm{~min}$ and cooling for $2 \mathrm{~h}$, was performed after dewaxing and rehydration to recover antigens. Endogenous peroxidase activity was blocked by incubation in $3 \%$ hydrogen peroxide at room temperature for $15 \mathrm{~min}$. Nonspecific binding was blocked by using $5 \%$ bovine serum albumin (BSA) at room temperature for $30 \mathrm{~min}$. Finally, the specimens were incubated overnight with antibodies against MMP-1 (cat. no. 10371-2-AP, 1:200; ProteinTech Group, Inc., Chicago, IL, USA); TIMP-1 (cat. no. 10753-1-AP, 1:100; ProteinTech Group, Inc.); TIMP-3 (cat. no. ab39184, 1:100; Abcam, Cambridge, UK) and the sections were stained by DAB the next day.

For further analyses, histological sections were clustered into fields of identical size. Subsequently, relative staining index was calculated by counting positively stained cells in 100 cells via Photoshop CS6 software (Adobe Systems, Inc., San Jose, CA, USA). For each group, a mean value of the 4 samples was calculated.

Immunohistofluorescence. Fresh tissue embedded in Tissue-Tek O.C.T. Compound (cat. no. 4583; Sakura Finetek USA, Inc., Torrance, CA, USA) was applied to obtain the frozen sections. After fixing in acetone for $30 \mathrm{~min}$, the sections were stained with PCNA (cat. no. 13110S, 1:100; Cell Signaling Technology, Inc., Danvers, MA, USA) and added an anti-rabbit IgG conjugated to Alexa Flour 405 (cat. no. R35551, 1:100; Invitrogen; Thermo Fisher Scientific, Inc., Waltham, MA, USA). Afterwards, the sections were observed with the help of a fluorescence microscope (N-STORM 4.0; Nikon Corporation, Tokyo, Japan) and the proliferation index was calculated by the ratio of positively stained cells to 100 cells.

Western blotting. The protein was extracted from the tissues in a lysis buffer with $0.2 \%$ protease inhibitor cocktail (Beyotime Institute of Biotechnology, Haimen, China). The lysis buffer solubilized protein was denatured in a loading buffer (Beyotime Institute of Biotechnology) at $95^{\circ} \mathrm{C}$ for $5 \mathrm{~min}$. The extracted $50 \mu \mathrm{g}$ of tissue was fractionated on a Bis-Tris gel and later was transferred to a PVDF membrane (both from Bio-Rad Laboratories, Inc., Hercules, CA, USA) by using semidry electroblotting. In order to block the non-specific binding, the membranes were incubated with 5\% BSA in TBST. Then, the membranes were incubated overnight at $4^{\circ} \mathrm{C}$ with antibodies against: MMP-2 (cat. no. ab37150, 1:1,000; Abcam); $\beta$-actin (cat. no. GTX11003, 1:1,000; GeneTex, Inc., Irvine, CA, USA); STAT3 (cat. no. ab68153, 1:2,000; Abcam); p-STAT3 (cat. no. ab76315, 1:1,000; Abcam); IL-6 (cat. no. ab9324, 1:500; Abcam); VCAM-1 (cat. no. ab134047, 1:2,000; Abcam); cleaved caspase-3 (cat. no. 9664T, 1:1,000; Cell Signaling Technology, Inc.). After washing for 5 times in TBST, the membranes were incubated with HRP-conjugated secondary antibodies (cat. nos. AS003 and AS014; ABclonal Biotech Co., Ltd., Woburn, MA, USA) at the dilution of 1:8,000 for $60 \mathrm{~min}$ at room temperature in TBST-BSA. The bands were detected by using a chemiluminescent peroxidase substrate (ECL; Amersham; GE Healthcare, Chicago, IL, USA) and exposed on a ChemDoc $^{\mathrm{TM}}$ MP (Bio-Rad Laboratories, Inc.).

Statistical analysis. The results were expressed as means \pm SD. Two value sets were compared by Student's t-test. For three or more values, one-way analysis of variance followed by Bonferoni post hoc test was used. The $\mathrm{P}<0.05$ was considered statistically significant. 


\section{Results}

Relative thickness of intima. Compared to allogenic transplantation groups, the intima of isogenic groups did not show an obvious thickening in the early postoperative period (within 7 days after transplantation). Analysis of specimens from allogenic groups has shown greater thickening of the intima than in the isogenic group $(\mathrm{P}<0.05$; Fig. 1$)$ in the late postoperative period. At the same time, the thickness of intima in the allogenic+SB-3CT treated group was lower compared to the allogenic group without treatment $(\mathrm{P}<0.05$; Fig. 1). As shown in Fig. 1, the mild hyperplasia in medial layers could be observed in the isogenic group on the 14th day after transplantation in contrast to allogenic groups where SMCs in medial layers have shown obvious proliferation and migration into the intima through the gap of elastic lamina.

Dynamic changes of MMPs/TIMPs. The MMP-1 and TIMP-1 showed the similar trend in the isogenic group, but after the 28th day decreased (Fig. 2). High expression of MMP-1 in allogenic groups was noticed on the 7 th day after the operation and peaked on the 14 th day $(\mathrm{P}<0.05$; Fig. $2 \mathrm{~A})$. The high expression of TIMP-1 was demonstrated for 14 days and peaked on the 28th day followed by a rapid drop on the 56th day $(\mathrm{P}<0.05$; Fig. 2B), however, the expression of TIMP-3 was only marginally expressed in the ECM of transplanted grafts and statistical difference was found only on the 7 th day ( $\mathrm{P}<0.05$; Fig. $2 \mathrm{C}$ ). The western blot analysis has shown relatively low expression of MMP-2 in the isogenic transplanted aorta and the expression of MMP-2 started to increase only after the 14th day and peaked on the 28th day followed by gradual reducing $(\mathrm{P}<0.05$; Fig. 3). In comparison to isogenic groups, MMP-2 was significantly upregulated on the 28th day $(\mathrm{P}<0.05$; Fig. 3$)$.

Inhibition of MMP-2 reduce aortic inflammation and apoptosis. We further looked into the parameters related to inflammation and apoptosis on the 7th day. Allogenic groups have shown more severe inflammation and apoptosis compared to the isogenic group as indicated by higher expression of p-STAT3, VCAM-1, proinflammatory cytokine IL-6, and cleaved-caspase 3 . However, the treatment of the allogenic group by SB-3CT after transplantation obviously reduced these damage-related parameters $(\mathrm{P}<0.05$; Fig. $4 \mathrm{~A}$ and $\mathrm{B})$. Immunohistofluorescence has shown that in the allogenic group there is the lowest proliferation index among the three groups while treatment with SB-3CT protects allografts from the injury by improving the proliferation $(\mathrm{P}<0.05$; Fig. $4 \mathrm{C}$ and D).

\section{Discussion}

Allograft vasculopathy (AV) is a progressive and diffuse intimal hyperplastic lesion of arteries that leads to gradual vessel narrowing and eventually to allograft ischemia (6). Blood vessels are regulated constantly by neurohormonal and hemodynamic changes. Chronic adaptation in vessels often occurs as a result of structural changes of their construction. MMPs are important enzymes of ECM degradation and they also regulate the biological activity of non-matrix substrates including cytokines, chemokines, receptors, growth factors

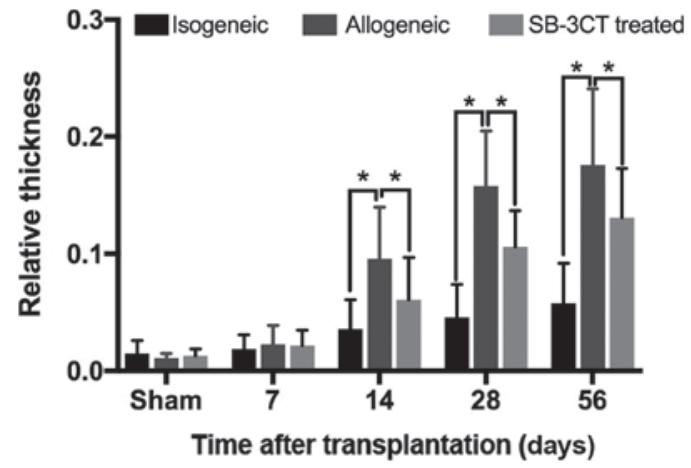

Figure 1. Relative thickness of intima. The intima of allografts showed obvious thickening on the 14th, 28th and 56th days after transplantation, however, the blockade of matrix metalloproteinase- 2 reduced the relative thickness of intima on the 14th day and on the 28th day after transplantation. ${ }^{*} \mathrm{P}<0.05$. SB-3CT, matrix metalloproteinase-2 inhibitor.

and cell adhesion molecules (3,4,7). Enzymatic activity is regulated at multiple levels and TIMPs participate in controlling the local activities of MMPs in the tissues (3).

Our study observed the expression of MMP-1, MMP-2, TIMP-1, and TIMP-3 in both allogenic and isogenic transplantation. Compared to the allografts, the isografts have demonstrated relatively lower expression of MMP-1, TIMP-1, and TIMP-3. Inhibition of MMP-2 reduced aortic inflammation and apoptosis by increasing the proliferation in allogenic groups. Vascular cell adhesion molecule 1 (VCAM-1) also konwn as cluster of differentiation 106 (CD106) is a protein mediates the adhesion of lymphocytes, monocytes, eosinophils to vascular endothelium and it also palys an important role in in the development of atherosclerosis $(8,9)$. Our supression of MMP-2 by SB-3CT in allogenic group lower the expression of VCAM-1 as well as the proinflammatory IL-6. IL-6 transduces signals via phosphorylation of STAT3 (pSTAT3), and STAT3 is a central regulator of inflammatory and immune responses (9). Thus, we might attribute the pathological changes to the imbalance of MMPs/TIMPs expression, which leads to the disorder of degradation of ECM and subsequently finish as arteriosclerosis of grafts. Vascular endothelial cells (ECs) were more susceptible to hypoxic-ischemic injuries in the early postoperative period. A series of cytokines such as bFGF and PDGF could be released by impaired ECs, that stimulate the expression of MMP-2 (10). Activated MMPs are able to degrade collagen, elastin, and other extracellular molecules (11). Endothelial inflammation (such as EC senescence, apoptosis, necrosis, and dysfunction) could be promoted by increased MMPs activity. MMP-1 enhances ECs senescence by p53 activation (12). MMP-2 cleaves the intercellular and cell-matrix junctions, including vascular endothelial-cadherin and $\beta$ - and $\gamma$-catenin that increase vascular wall permeability $(13,14)$. Some studies suggest that activated MMPs function as growth factors of VSMCs in vitro. The MMP-mediated VSMC proliferation in vivo contributes to arterial intimal-medial thickening (IMT) $(15,16)$.

In the late postoperative period, the increased ratio of $\mathrm{CD}^{+} / \mathrm{CD}^{+} \mathrm{T}$ cells and enormous inflammatory cytokines can induce the gene transcription of TIMP-1 $(1,17)$. TIMP-1 regulates the activity of MMPs-enzymes, which are involved in the degradation of the ECM (18). This mechanism will cause the 

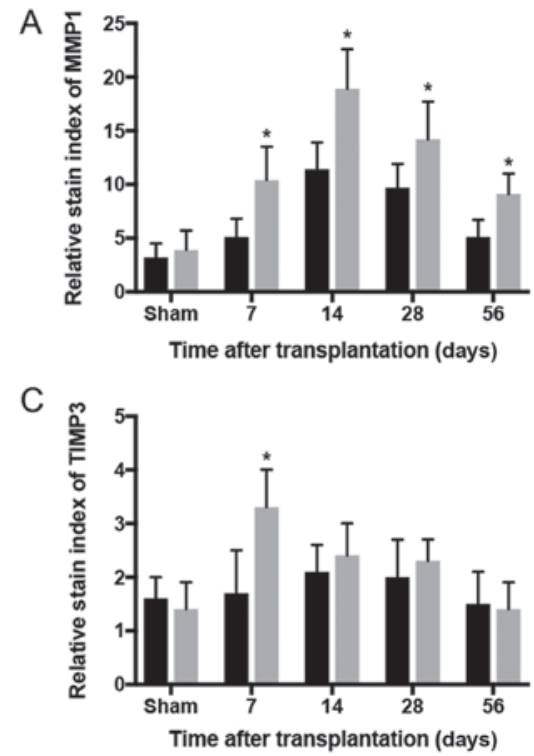

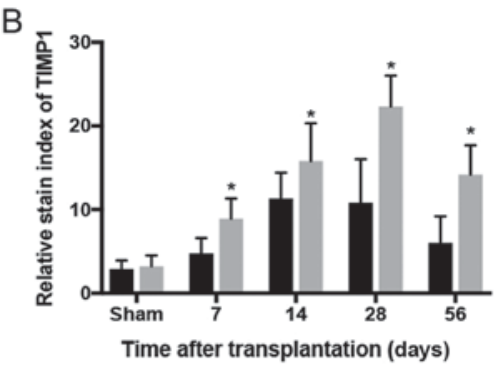

Isogeneic

Allogeneic

Figure 2. Relative staining index of MMP-1, TIMP-1, and TIMP-3 in isogenic and allogenic groups. Relative high staining index of (A) MMP-1 and (B) TIMP-1 was observed for 14 days, however, the statistical difference of (C) TIMP-3 was found only on the 7 th day. "P<0.05 vs. the isogenic group. MMP, matrix metalloproteinase; TIMP, tissue inhibitor of metalloproteinases.
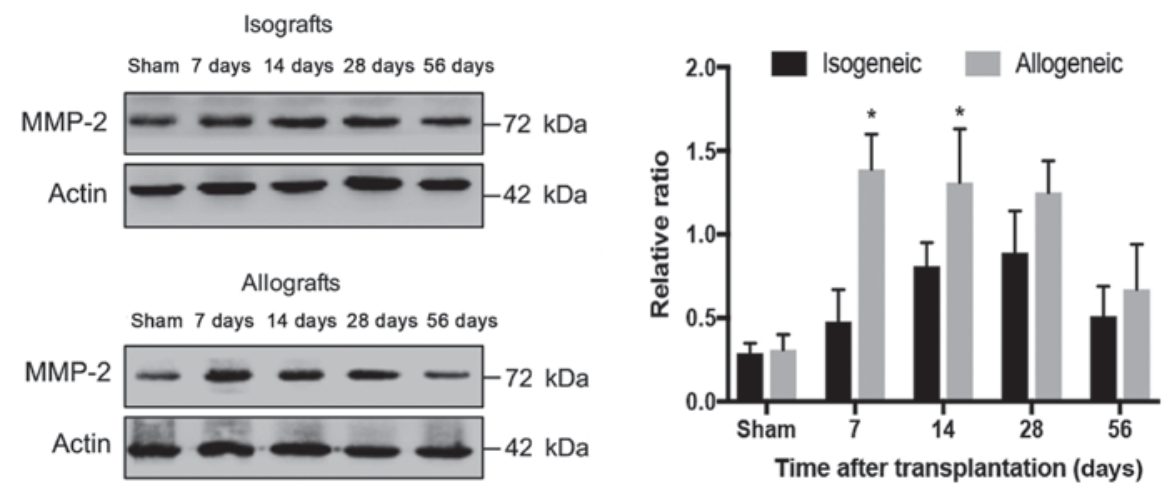

Figure 3. Western blotting of MMP-2 expression in the isogenic and allogenic groups. Western blotting determined that the expression of MMP-2 started to increase after the 7th day of allotransplanation, while in the isogenic transplanted aorta, MMP-2 remained relatively steady and started to increase only after 14th day followed by gradually reduced expressions. " $\mathrm{P}<0.05$ vs. the isogenic group. MMP, matrix metalloproteinase.
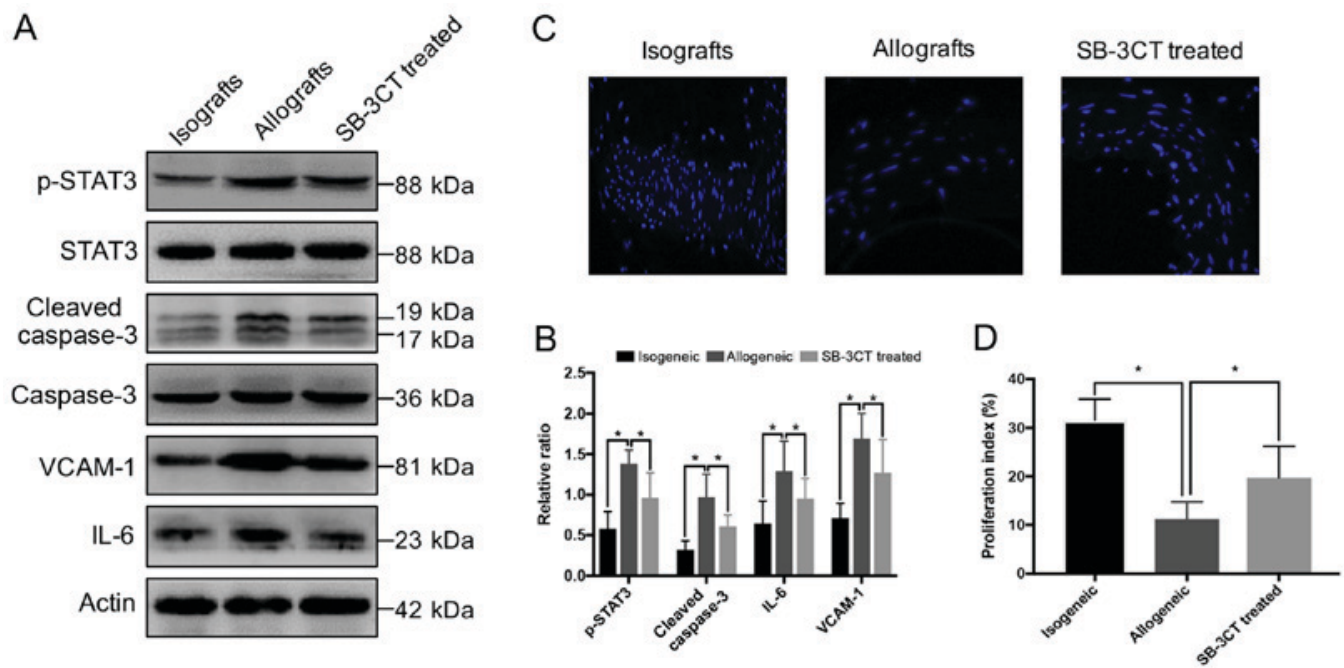

Figure 4. SB-3CT treatment alleviated aortic injury. (A and B) Representative Western blot images and statistical analysis in three groups on injury-related parameters; (C and D) representative immunofluorescence of PCNA and statistical analysis in three groups; A potent inhibitor of matrix metalloproteinase-2 protected the allografts from inflammation and apoptosis by increasing proliferation. ${ }^{*} \mathrm{P}<0.05$. Magnification, $\mathrm{x} 400$. STAT, signal transducer and activator of transcription; VCAM, vascular cell adhesion protein; IL, interleukin; SB-3CT, matrix metalloproteinase-2 inhibitor. 
accumulation of collagen in the extracellular space and further will lead to arteriosclerosis. The growing evidence from the past decade clearly indicates that TIMPs have additional biological activities by acting as signaling molecules (19). The cytokine-like activities of TIMPs include the modulation of cell proliferation, apoptosis, differentiation, and angiogenesis (20) that contribute to the formation of atherosclerosis. Interestingly, the expression of TIMP-3 was low in both groups. TIMPs are specific endogenous inhibitors that bind MMPs in a 1:1 stoichiometry, however, the inhibitory properties of TIMP-3 are different from the rest TIMPs, because TIMP-3 inhibits ADAMTS (a disintegrin-like and metalloproteinase domain with thrombospondin-type 1 motifs) (21). The recent biochemical data have clarified some aspects of the relationship between ADAMTS proteins and fibrillin microfibrils (22) and we suspect that this might be an important reason for arteriosclerosis.

Our present study only showed a relativly long term (up to 56 days) of posttransplantation. In future, we would like to perform a real long-term data (months, perhaps years) using an in vivo model where animals receive immunosupression therapy to study more about inflammatory cell infiltration and EC activation to support the results of the present study.

In conclusion our study has revealed the imbalance of MMPs/TIMPs in isogenic and allogenic orthotopic aorta transplantation and inhibition of MMP-2 reduced the aortic inflammation and apoptosis by increasing the proliferation in the allogenic group. It is assumed that this imbalance leads to the disturbance of the ECM and is related to the posttransplantation damage.

\section{Acknowledgements}

Not applicable.

\section{Funding}

This study was supported by the National Natural Science Foundation of China (grant no. 81370577).

\section{Availability of data and materials}

All data generated or analyzed during this study are included in this published article.

\section{Authors' contributions}

SDW and MHW conceived and designed the experiments. SDW, DC and XSX performed the experiments. HB and HW analyzed the data. MHW contributed reagents, materials and instructions for the model establishment. SDW and MHW wrote the paper.

\section{Ethics approval and consent to participate}

The study was approved by the Animal Care and Use Committee of Second Affiliated Hospital of Chongqing Medical University (Chongqing, China).

\section{Consent for publication}

Not applicable.

\section{Competing interests}

The authors declare that they have no competing interests.

\section{References}

1. von Rossum A, Laher I and Choy JC: Immune-mediated vascular injury and dysfunction in transplant arteriosclerosis. Front Immunol 5: 684, 2014.

2. Rahmani M, Cruz RP, Granville DJ and McManus BM: Allograft vasculopathy versus atherosclerosis. Circ Res 99: 801-815, 2006

3. Visse R and Nagase H: Matrix metalloproteinases and tissue inhibitors of metalloproteinases: Structure, function, and biochemistry. Circ Res 92: 827-839, 2003.

4. Galis ZS and Khatri JJ: Matrix metalloproteinases in vascular remodeling and atherogenesis: The good, the bad, and the ugly. Circ Res 90: 251-262, 2002.

5. Sommer W, Knöfel AK, Izykowski N, Oldhafer F, Avsar M, Jonigk D, Warnecke G and Haverich A: Physical exercise reduces transplant arteriosclerosis in a mouse aorta transplantation model. J Thorac Cardiovasc Surg 149: 330-337, 2015.

6. Mitchell RN: Learning from rejection: What transplantation teaches us about (other) vascular pathologies. J Autoimmun 45: 80-89, 2013.

7. Dimas G, Iliadis F and Grekas D: Matrix metalloproteinases, atherosclerosis, proteinuria and kidney disease: Linkage-based approaches. Hippokratia 17: 292-297, 2013.

8. Kitamura H, Ohno Y, Toyoshima Y, Ohtake J, Homma S, Kawamura H, Takahashi N and Taketomi A: Interleukin-6/STAT3 signaling as a promising target to improve the efficacy of cancer immunotherapy. Cancer Sci 108: 1947-1952, 2017.

9. Calautti E, Avalle L and Poli V: Psoriasis: A STAT3-centric view. Int J Mol Sci 19: pii: E171, 2018.

10. Solomon A, Li DQ, Lee SB and Tseng SC: Regulation of collagenase, stromelysin, and urokinase-type plasminogen activator in primary pterygium body fibroblasts by inflammatory cytokines. Invest Ophthalmol Vis Sci 41: 2154-2163, 2000.

11. Lakatta EG: The reality of aging viewed from the arterial wall. Artery Res 7: 73-80, 2013.

12. Struewing IT, Durham SN, Barnett CD and Mao CD: Enhanced endothelial cell senescence by lithium-induced matrix metalloproteinase-1 expression. J Biol Chem 284: 17595-17606, 2009.

13. Wu WB and Huang TF: Activation of MMP-2, cleavage of matrix proteins and adherens junctions during a snake venom metalloproteinase-induced endothelial cell apoptosis. Exp Cell Res 288: 143-157, 2003.

14. Shapiro S, Khodalev O, Bitterman H, Auslender R and Lahat N: Different activation forms of MMP-2 oppositely affect the fate of endothelial cells. Am J Physiol Cell Physiol 298: C942-C951, 2010.

15. Xiao Q, Zhang F, Grassia G, Hu Y, Zhang Z, Xing Q, Yin X, Maddaluno M, Drung B, Schmidt B, et al: Matrix metalloproteinase- 8 promotes vascular smooth muscle cell proliferation and neointima formation. Arterioscler Thromb Vasc Biol 34: 90-98, 2014.

16. Austin KM, Nguyen N, Javid G, Covic L and Kuliopulos A: Noncanonical matrix metalloprotease-1-protease-activated receptor-1 signaling triggers vascular smooth muscle cell dedifferentiation and arterial stenosis. J Biol Chem 288: 23105-23115, 2013.

17. Hsieh JL, Shiau AL, Lee CH, Yang SJ, Lee BO, Jou IM, Wu CL, Chen SH and Shen PC: $\mathrm{CD}^{+} \mathrm{T}$ cell-induced expression of tissue inhibitor of metalloproteinses-1 exacerbated osteoarthritis. Int J Mol Sci 14: 19951-19970, 2013.

18. Raffetto JD and Khalil RA: Matrix metalloproteinases and their inhibitors in vascular remodeling and vascular disease. Biochem Pharmacol 75: 346-359, 2008.

19. Ries C: Cytokine functions of TIMP-1. Cell Mol Life Sci 71: 659-672, 2014.

20. Lambert E, Dassé E, Haye B and Petitfrère E: TIMPs as multifacial proteins. Crit Rev Oncol Hematol 49: 187-198, 2004.

21. Kashiwagi M, Tortorella M, Nagase H and Brew K: TIMP-3 is a potent inhibitor of aggrecanase 1 (ADAM-TS4) and aggrecanase 2 (ADAM-TS5). J Biol Chem 276: 12501-12514, 2001.

22. Hubmacher D and Apte SS: ADAMTS proteins as modulators of microfibril formation and function. Matrix Biol 47: 34-43, 2015. 